\title{
Incidentally Found Aorto-Pulmonary Middle Mediastinal Hypervascular Mass on Pulmonary CT Angiography in a Covid 19 patient
}

Nu Nu Win ${ }^{1}$, Berrin Erok ${ }^{2}$

${ }^{1}$ Department of Radiology, Medicana Bahcelievler Hospital, İstanbul, Turkey.

${ }^{2}$ Universtiy of Health Sciences, Department of Radiology, Prof Dr Cemil Tascioglu City Hospital, İstanbul, Turkey.

*Corresponding Author: Berrin Erok, Universtiy of Health Sciences, Department of Radiology, Prof Dr Cemil Tascioglu City Hospital, İstanbul, Turkey.

Received date: Febuary 06, 2021; Accepted date: March 08, 2021; Published date: March 11, 2021

Citation: Nu N Win, B Erok. (2021) Incidentally Found Aorto-Pulmonary Middle Mediastinal Hypervascular Mass on Pulmonary CT

Angiography in a Covid 19 patient. International Journal of Clinical Case Reports and Reviews. 6(4); DOI:10.31579/2690-4861/114

Copyright: (C) 2021 Berrin Erok, This is an open-access article distributed under the terms of the Creative Commons Attribution License, which permits unrestricted use, distribution, and reproduction in any medium, provided the original author and source are credited.

\begin{abstract}
Paragangliomas (PGs) are rare neuroendocrine tumors arising from paraganglia, clusters of neuroendocrine cells scattered throughout the body. Mediastinal paragangliomas represent less than $2 \%$ of all paragangliomas and less than $0.3 \%$ of the mediastinal tumors. These tumors may secrete catecholamines, however in up to $50 \%$ of cases they are nonfunctional and are diagnosed incidentaly or with symptoms of mass effcet to the adjacent structures. They should be considered in the differential diagnosis of hypervascular mediastinal masses. The typical radiological features are very guiding in the diagnosis and the management of the patient. We aimed to present a case of a hypervascular middle mediastinal mass incidentally found in a 69 year old woman and diagnosed with aortopulmonary nonfunctional PG radiologically.
\end{abstract}

Keywords: mediastinal paraganglioma; glomus tumor; hypervascular mediastinal mass

\section{Introduction}

Paragangliomas (PGs), also sometimes called as glomus tumors, are rare neuroendocrine tumors arising from paraganglia, which are clusters of neuroendocrine cells scattered throughout the body with the largest cluster found in the adrenal medulla. These cells are closely related to the autonomic nervous system, with either parasympathetic or sympathetic function. While the parasympathetic PGs are generally nonsecretory and present usually with mass-effect such as cranial nerve palsies, the symphathetic paragangliomas tend to be functional and secrete catecholamines resulting in presentations with palpitations, hypertension, headaches, hyperhidrosis and diabetes which resolves after the complete resection of the PG [1,2] Symphathetic PGs can be intraadrenal which are called as pheochromocytoma or extra-adrenal which can be located in the abdomen or thorax (mediastinal PG) [3]. Mediastinal PGs represent less than $2 \%$ of all PGs and less than $0.3 \%$ of the mediastinal tumors [4]. We present a case of a hypervascular middle mediastinal mass incidentally found in a 69 year old woman and diagnosed with aortopulmonary nonfunctional PG radiologically.

\section{Case Resentation}

A 69 year old female patient presented with cough, mild fever and chest pain. She had recent history of Covid-19 pneumonia about 8 months ago. Her laboratory results revealed increased level of D-dimer and Ferritin with slight increase in WBC count and CRP. There were no known any other diseases or primary malignancy. We performed IV contrast enhanced chest computed tomography $[\mathrm{CT}]$ for her presenting symptoms, which revealed a well defined middle mediastinal mass between left pulmonary artery and the descending aorta showing intermediate soft tissue density on precontrast images without any calcifications (fig. 1a). The size of the mass was abouut $3 \times 3 \mathrm{~cm}$ and showed no enhancement on pulmonary arterial phase images (fig.1b) and heterogeneously intense enhancement on early arterial phase images (fig. 1c) with multiple serpentine tubular enhancing vasculature within the lesion. There were no accompanying pulmonary parenchymal nodules/masses, pathologic mediastinal lymph nodes or any parenchymal infiltration. Although we did not concerned malignancy and metastases, they were ruled out by positron emission CT (PET-CT). Our main differential diagnosis included mediastinal PG and mediastinal hemangioma. Due to the characteristic location of the mass in the middle mediastinum, the heterogenous serpentine contrast enhancement pattern immediately at the early arterial phase CT images and the lack of phlebolit on nonenhanced images, our initial diagnosis was mediastinal PG. Although there were no symptoms suggesting increased catecholamine secretion and her 24 hour urine vanillyl mandelic acid level was within normal limits, excision of the mass was recommended rather than biopsy because of hypervascularity and close proximity of the lesion to the great vessels. However, she refused operation due to her asymptomatic course and wanted to be followed up.

*informed consent has been taken from the patient. 

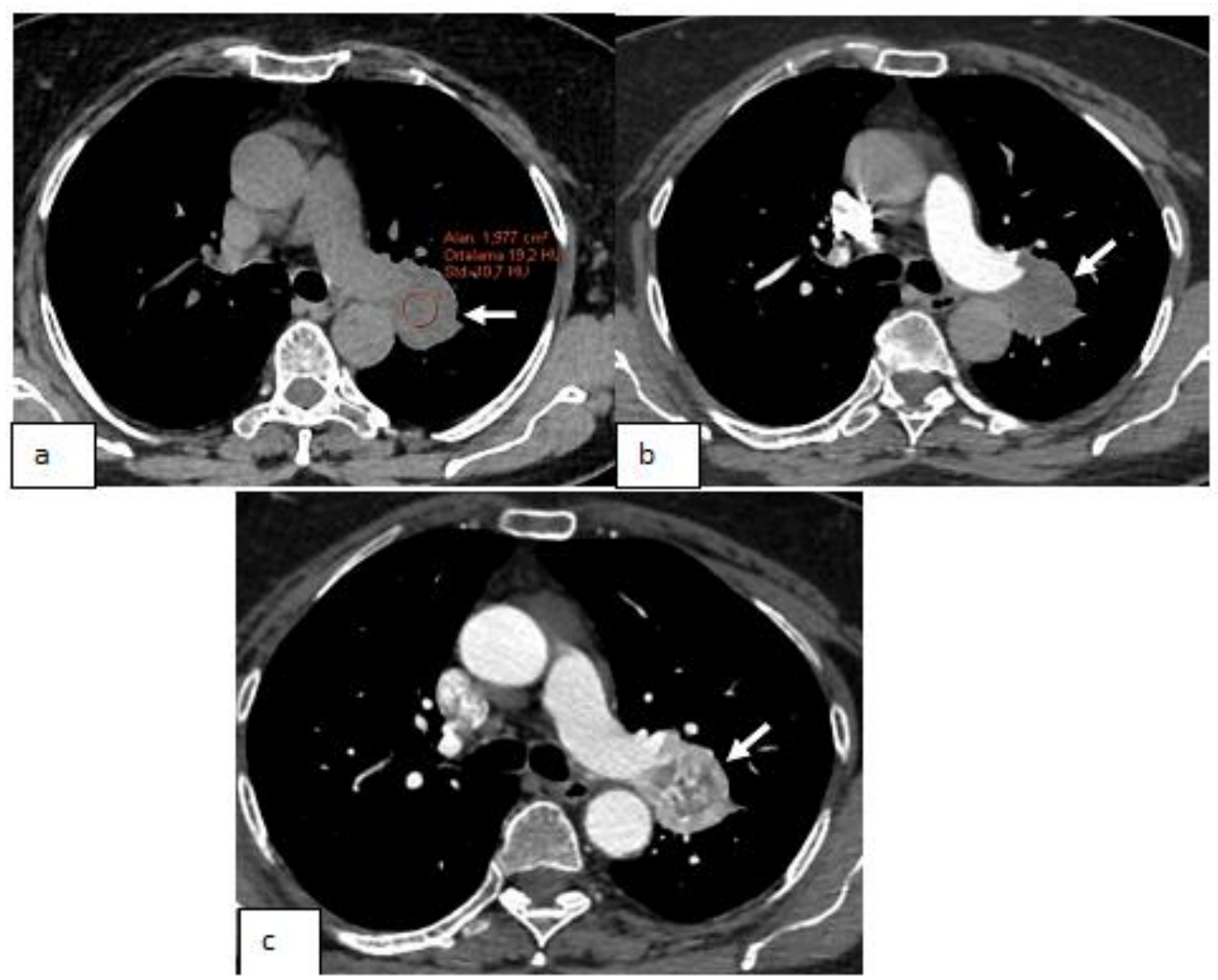

Figure 1a: Axial precontrast CT image and b,c Axial Pulmonary CTA images showing a well-defined middle mediastinal mass between left pulmonary artery and the descending aorta with intermediate soft tissue density on precontrast (a, arrow) without any calcifications and pulmonary arterial phase images (b, arrow) without enhancement. Heterogeneously intense enhancement on early arterial phase images with multiple serpentine tortious tubular enhancing vasculature within the lesion is demonstrated (c, arrow).

\section{Discussion}

Mediastinal PGs are rare tumors that can arise from two major clusters of sympathetic paraganglion cells including aortosympathetic paraganglia in the posterior mediastinum [paravertebral] and aortopulmonary paraganglia in the middle mediastinum [great vessels of the chest]. Additionally, although extremelly rare the third type of the mediastinal PG is cardiac PG [5]. These tumors may secrete catecholamines, however in up to $50 \%$ of cases they are nonfunctional. Most patients are asymptomatic and the diagnosis is incidental $[1,6]$. Others may present with symptoms of local mass effect to the adjacent structures resulting in hoarseness, dysphagia, shortness of breath and chest pain [1,7]. In addition, PGs may develop distant metastases occuring most commonly to lymph nodes, liver, lung, and bone. Since there are no reliable histological marker for their malignant potential, the presence of metastases can be the only evidence for malignancy [8]. When the primary diagnostic concern is PG, biopsy is contindicated until biochemical screening is negative for catecholamine excess PGs are seen in a characteristic location in the paraaortic region of the middle mediastinum or paravertebral region of the posterior mediastinum corresponding to major cluster of sympathetic ganglion cells [3]. On nonenhanced CT they have typically intermediate density and show heterogenous intense enhancement following contrast administration. On MRI, they are usually markedly hyperintense on T2-w images representing light bulb appearance [9]. Larger ones display salt-and- pepper appearance referring to slow flow within dilated vascular structures or foci of hemorrhages (salt) and flow voids within high flow vasculature [pepper] [7]. Following contrast administration due to the presence of rich capillary network arising generally from internal mammmary artery, they demonstrate intense heterogenous, prolonged enhancement and delayed washout on dynamic imagings [9]. Differential diagnoses include mediastinal hemangioma and hypervascular metastases such as from RCC [10]. In hypervascular mediastinal metastasis, there is usually a known previous history of primary malignancy and the lesions are usually more than one in number with frequently accompanying pulmonary parenchymal involvement. In our patient there were no history of malignancy and thoracoabdominal imagings revealed no additional pathology. Therefore, our main differential diagnosis was mediastinal hemangioma. However, the middle mediastinum is the least common location for mediastinal hemangiomas with more than $50 \%$ of mediastinal hemangiomas being located in the anterior mediastinum [11, 12]. Phlebolithes can be characteristically demonstrated on nonenhanced images in cases of mediastinal hemangiomas [13]. In addition, although not always, hemangiomas, if larger than $1.5 \mathrm{~cm}$ in diameter are expected to demonstrate peripheral nodular discontinuous enhancement on early arterial phase images and progressive centripetal fill-in on delayed phase images over time. With the consideration of excessive ionising radiation we did not perform dynamic contrast enhanced CT. In our patient, due to the characteristic location of the mass in the middle mediastinum, the 
heterogenous serpentine contrast enhancement pattern immediately at the early arterial phase CT images rather than only peripheral pathcy nodular enhancement and the lack of phlebolit on nonenhanced images, we diagnosed radiologically the lesion as mediastinal PG. Although the PG has been showed to be nonfunctional, we have recommended surgical excision rather than biopsy due to the hypervascularity of the lesion and its close proximity to the great vessels. She refused operation due to her asymptomatic course and wanted to be followed up.

\section{Conclusion}

Mediastinal PGs are rare lesions which constitute less than $0.3 \%$ of the mediastinal tumors. However, they should be considered in the differential diagnosis of hypervascular mediastinal masses. The typical radiological features are very guiding in the diagnosis and the management of the patient

\section{References}

1. Wald O, Shapira OM, Murar A, et al. (2010) Paraganglioma of the mediastinum: challenges in diagnosis and surgical management. $\mathrm{J}$ Cardiothorac Surg.5:19.

2. Miura K, Kobayashi N, Satomi H. (2020) Surgical resection of a middle mediastinal paraganglioma that caused diabetes. Surg Case Rep. 30;6(1):241.

3. Lam AK. Update on Adrenal Tumours in 2017 World Health Organization (WHO) of Endocrine Tumours. (2017) Endocrine pathology. 28 (3): 213-227.

4. Ayadi-Kaddour A, Braham E, Ismail O, et al. Posterior mediastinal paragangliomas: a report of three patients with peculiar tumours. Respirology. 14:459-461.
5. Grebenc ML, Rosado de Christenson ML, Burke AP, Green CE, Galvin JR. Primary cardiac and pericardial neoplasms: radiologicpathologic correlation. Radiographics : a review publication of the Radiological Society of North America, Inc. 20 (4):1073-1103.

6. Young WF Jr. (2006) Paragangliomas: clinical overview. Ann N Y Acad Sci. 1073:21-29.

7. Balcombe J, Torigian DA, Kim W, Miller WT Jr. (2007) Crosssectional imaging of paragangliomas of the aortic body and other thoracic branchiomeric paraganglia. AJR Am J Roentgenol. 188(4):1054-1058.

8. Fliedner SM, Lehnert H, Pacak K. Metastatic paraganglioma. (2010) Seminars in oncology. 37 (6): 627-637.

9. Nakazono T, White CS, Yamasaki F, et al. (2011) MRI findings of mediastinal neurogenic tumors. AJR Am J Roentgenol. 197(4):643-652.

10. Drucker EA, Mcloud TC, Dedrick CG et-al. (1987) Mediastinal paraganglioma: radiologic evaluation of an unusual vascular tumor. AJR Am J Roentgenol. 148 (3):521-522.

11. Yasunori S, Masakazu K, Hidenori K, Fumihiro S, Chie U, Sadanori T. (2013) A case of middle mediastinal cavernous hemangioma. Gen Thorac Cardiovasc Surg.

12. Xianqi Cai, Chunquan Liu, and Yong Cui. (2020) A case of middle mediastinal cavernous hemangioma. Thorac Cancer. 11(3): 789-792.

13. Li SM, Hsu HH, Lee SC, Gao HW, Ko KH. (2017) Mediastinal hemangioma presenting with a characteristic feature on dynamic computed tomography images. J Thorac Dis. 9 (5):412-415.
This work is licensed under Creative Commons Attribution 4.0 License

To Submit Your Article Click Here: Submit Manuscript

DOI: $10.31579 / 2690-4861 / 114$

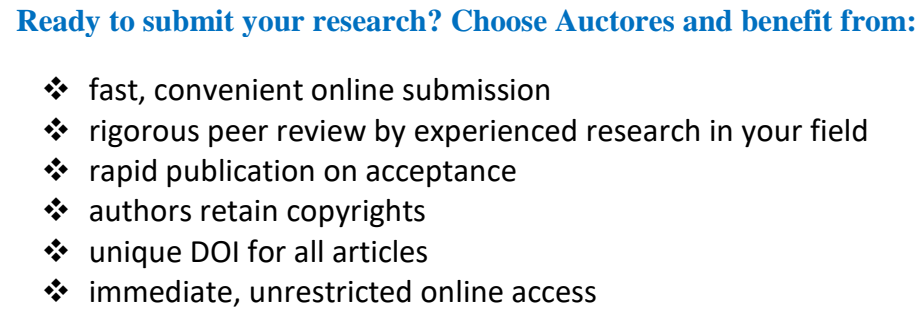

At Auctores, research is always in progress.

Learn more www.auctoresonline.org/journals/international-journal-ofclinical-case-reports-and-reviews 